\title{
Role of histone deacetylase activity in the developing lateral line neuromast of zebrafish larvae
}

\begin{abstract}
Yingzi He${ }^{1,2}$, Honglin $\mathrm{Mei}^{1}$, Huiqian $\mathrm{Yu}^{1}$, Shan $\mathrm{Sun}^{1}$, Wenli $\mathrm{Ni}^{1}$ and Huawei $\mathrm{Li}^{1,2,3}$
Histone deacetylases are involved in many biological processes and have roles in regulating cell behaviors such as cell cycle entry, cell proliferation and apoptosis. However, the effect of histone deacetylases on the development of hair cells (HCs) has not been fully elucidated. In this study, we examined the influence of histone deacetylases on the early development of neuromasts in the lateral line of zebrafish. Hair cell development was evaluated by fluorescent immunostaining in the absence or presence of histone deacetylase inhibitors. Our results suggested that pharmacological inhibition of histone deacetylases with inhibitors, including trichostatin A, valproic acid and MS-275, reduced the numbers of both HCs and supporting cells in neuromasts. We also found that the treatment of zebrafish larvae with inhibitors caused accumulation of histone acetylation and suppressed proliferation of neuromast cells. Real-time PCR results showed that the expression of both p21 and p27 mRNA was increased following trichostatin A treatment and the increase in p53 mRNA was modest under the same conditions. However, the expression of p53 mRNA was significantly increased by treatment with a high concentration of trichostatin A. A high concentration of trichostatin A also led to increased cell death in neuromasts as detected in a TUNEL assay. Moreover, the nuclei of most of these pyknotic cells were immunohistochemically positive for cleaved caspase-3. These results suggest that histone deacetylase activity is involved in lateral line development in the zebrafish and might have a role in neuromast formation by altering cell proliferation through the expression of cell cycle regulatory proteins.
\end{abstract}

Experimental \& Molecular Medicine (2014) 46, e94; doi:10.1038/emm.2014.18; published online 9 May 2014

Keywords: hair cell; histone deacetylases; neuromast; supporting cell; zebrafish

\section{INTRODUCTION}

The zebrafish lateral line system comprises a set of rosette-like sensory organs, called neuromasts, that are located on the surface of the head and the body and are used to detect directional water flow. ${ }^{1}$ The neuromast is made of a group of hair cells (HCs) in the center and supporting cells on the periphery. HCs in the neuromast are closely related to the mammalian inner ear sensory HCs in terms of both morphology and function. ${ }^{2,3}$ The use of the zebrafish lateral line as a way of studying hair cell development offers several distinct advantages. For example, because the larva is optically transparent, live imaging can be used to follow early embryogenesis. In addition, due to their external location, the HCs can be readily stained by fluorescent dyes for experimental detection. Another advantage of using the zebrafish lateral line system is the ability of the neuromast to produce newly differentiated HCs primarily from the continuous proliferation of supporting cells after the original HCs have been destroyed. ${ }^{4}$ Therefore, neuromasts provide a powerful tool to investigate mechanosensory HC development and to screen for potential ototoxic and otoprotective drugs. ${ }^{5}$

The normal development, survival and function of the lateral line neuromast in zebrafish depend on coordinated and strictly regulated gene expression. Epigenetic mechanisms have key roles in eukaryotic gene expression by affecting posttranslational modifications such as phosphorylation, ubiquitination, sumoylation, acetylation and methylation of conserved lysines in the N-terminal tails of the core histones. ${ }^{6,7}$ Recently, chromatin remodeling and transcriptional regulation by posttranslational histone modification have attracted increasing attention due to their pivotal roles in many biological processes, especially those related to hearing. ${ }^{8}$ Acetylation and deacetylation of histones are the most common histone modifications and are governed by two classes of enzymes,

\footnotetext{
${ }^{1}$ Department of Otology Skull Base Surgery, Hearing Research Institute, Eye and ENT Hospital of Shanghai Medical School, Fudan University, Shanghai, China; ${ }^{2}$ Institutes of Biomedical Sciences, Fudan University, Shanghai, China and ${ }^{3}$ State Key Laboratory of Medical Neurobiology, Fudan University, Shanghai, China

Correspondence: Professor H Li, Department of Otolaryngology, Eye and ENT Hospital, Fudan University, Room 611, Building 9, No. 83, Fenyang Road, Xuhui District, Shanghai 200031, China.

E-mail: hwli@shmu.edu.cn
}

Received 27 August 2013; revised 7 December 2013; accepted 21 December 2013 
histone acetyltransferases (HATs) and histone deacetylases (HDACs).

HATs and HDACs are involved in many developmental processes by modulating chromatin structure, ${ }^{9}$ and the specific roles of HDACs in controlling neuronal development and specification have attracted attention over the past few years. Some reports have indicated that HDAC1 is a critical regulator of neuronal differentiation and is required for the transformation of neural precursors into neurons during the development of zebrafish and mice. ${ }^{10-12}$ Cells lacking HDAC1 and HDAC2 are unable to differentiate into mature neurons. Recently, a variety of HDAC inhibitors have been developed, and these have been shown to shift the balance between HAT and HDAC activities toward histone hyperacetylation and promote growth arrest, differentiation and even apoptosis in certain cancer cells. ${ }^{13}$ It has also been suggested that HDAC inhibitors can be used to correct some deficiencies associated with human brain disorders. ${ }^{14,15}$

Previous studies with avian utricles showed that HDACs assist in the regulation of supporting cell proliferation in sensory epithelia. ${ }^{16}$ However, a detailed understanding of whether and how HDAC activity is connected to $\mathrm{HC}$ development is still lacking. Considering the similarity of HCs in the neuromast of the zebrafish lateral line to those in the mammalian inner ear, a better understanding of the function of HDAC activity on the development of lateral line HCs is of interest in terms of research into hearing-related mechanisms in mammals. In this study, we analyzed the effect of HDACs on the development of lateral line neuromast HCs by exposing zebrafish larva to HDAC inhibitors. We demonstrated that pharmacological inhibition of HDACs reduced the numbers of $\mathrm{HCs}$ and supporting cells in neuromasts. We also showed that HDAC inhibitors markedly decreased the cell proliferation in the developing neuromasts. These results suggest that HDAC activity is involved in neuromast development and might be a positive regulator in the formation of HCs and SCs in the lateral line.

\section{MATERIALS AND METHODS}

\section{Zebrafish embryos and larvae}

Zebrafish embryos were obtained from the natural spawning of wildtype adults and maintained in our facility according to standard procedures. The Brn3c:mGFP transgenic line was obtained from Professor Zhengyi Chen, our collaborator at Harvard University. Zebrafish larvae were staged according to the method discussed by Kimmel et al. ${ }^{17}$ Ages of embryos are described as days post fertilization (dpf).

\section{Pharmacological treatments}

HDAC inhibitors - valproic acid (VPA, Sigma), Trichostatin A (TSA, Sigma), and MS-275 (Calbiochem)—were dissolved either in ddH2O (VPA) or DMSO (TSA, MS-275) at stock concentrations of $200 \mathrm{~mm}$, $500 \mu \mathrm{M}$ and $10 \mathrm{~mm}$, respectively, and then diluted to the final concentrations in fresh egg water. Dose-response data were obtained by treating $3 \mathrm{dpf}$ larvae for 2 days with VPA $(50,100$ and $150 \mu \mathrm{M})$, TSA $(0.05,0.1$ and $0.2 \mu \mathrm{M})$ or MS-275 $(5,10$ and $15 \mu \mathrm{M})$ after which the larvae were fixed. In all experiments, control groups were maintained in parallel under the same conditions, including the DMSO used with TSA and MS-275, but without HDAC inhibitors.

\section{FM1-43FX labeling}

For staining of functional HCs within the neuromasts, the mechanotransduction marker FM1-43FX (Invitrogen, Carlsbad, CA, USA; F-35355) was applied at a concentration of $3 \mu \mathrm{M}$ to live $5 \mathrm{dpf}$ larvae for $45 \mathrm{~s}$ in the dark. After quickly rinsing three times with fresh egg water, the larvae were anesthetized in MS-222 (Sigma-Aldrich, St Louis, MO, USA) and fixed with $4 \%$ paraformaldehyde (PFA). The labeled HCs of the first four neuromasts along the body, L1 to L4, were recorded on one side of each fish (Figure 1d).

\section{Cell proliferation and analysis}

Proliferating cells in the lateral line neuromasts were labeled by adding $10 \mathrm{~mm}$ 5-bromo-2-deoxyuridine (BrdU; Sigma) to the fresh egg water for 2 days at $28.5^{\circ} \mathrm{C}$. Larvae were fixed with $4 \%$ PFA overnight at $4{ }^{\circ} \mathrm{C}$, and $\mathrm{BrdU}$ incorporation was detected by fluorescent immunostaining. The fixed larvae were washed several times in PBS containing $0.5 \%$ Triton X-100 (PBT-2) and placed into $2 \mathrm{~N} \mathrm{HCl}$ for $0.5 \mathrm{~h}$ at $37^{\circ} \mathrm{C}$. Larvae were blocked in $10 \%$ normal goat serum for $1 \mathrm{~h}$ at room temperature and incubated with the monoclonal primary anti-BrdU antibody (1:200 dilution) overnight at $4{ }^{\circ} \mathrm{C}$. The next day, larvae were washed three times for $10 \mathrm{~min}$ each with PBT-2 and incubated with the secondary antibody for $1 \mathrm{~h}$ at $37^{\circ} \mathrm{C}$. Images from the labeled sections were captured with a CCD spot camera, and images from the whole cochleae were captured with a Leica confocal fluorescence microscope (TCS SP5, Leica, Wetzlar, Germany).

\section{Immunohistochemistry}

For immunohistochemistry analysis, $5 \mathrm{dpf}$ larvae were fixed in $4 \%$ PFA and were permeabilized with $0.5 \%$ PBT-2 for $30 \mathrm{~min}$ followed by incubation in blocking solution for $1 \mathrm{~h}$. The following antibodies were used as primary antibodies: anti-myosin VI (1:200); anti-Sox2 (1:200); anti-acetylated histone H4 (1:400); anti-acetylated histone H3 (1:400); anti-cleaved caspase-3 (1:200). The embryos were washed three times with PBS and incubated with secondary antibodies to detect primary antibodies. Nuclei were labeled with DAPI for $20 \mathrm{~min}$ at room temperature.

\section{Western blot analysis}

Total protein was isolated from $5 \mathrm{dpf}$ larvae with the AllPrep DNA/ RNA/Protein Mini Kit (Qiagen, Hilden, Germany) according to the manufacturer's instructions. Protein concentrations were measured using a BCA protein kit, and samples were separated by $12 \%$ SDS-PAGE. After electrophoresis, the proteins were transferred onto PVDF membranes (Immobilon-P; Millipore, Bedford, MA, USA) that were blocked with $5 \%$ nonfat dried milk in TBST $(50 \mathrm{~mm}$ Tris- $\mathrm{HCl}$ ( $\mathrm{pH}$ 7.4), $150 \mathrm{~mm} \mathrm{NaCl}, 0.1 \%$ Tween-20) for $1 \mathrm{~h}$ at room temperature. After washing, anti-acetylated histone H3 (1:1000), anti-acetylated histone H4 (1:1000), anti-histone H3 (1:1000) and anti-histone H4 (1:1000) primary antibodies were added to blocking buffer overnight at $4{ }^{\circ} \mathrm{C}$. The membranes were washed three times $(10 \mathrm{~min}$ each) with TBST.

\section{Real-time RT-PCR analysis of p21, p27 and p53 mRNA}

Total RNA was extracted from zebrafish larvae using Trizol reagent (Invitrogen) according to the manufacturer's protocol. Reverse transcription was performed with Superscript III (Invitrogen) following the manufacturer's instructions. The cDNA was analyzed by 


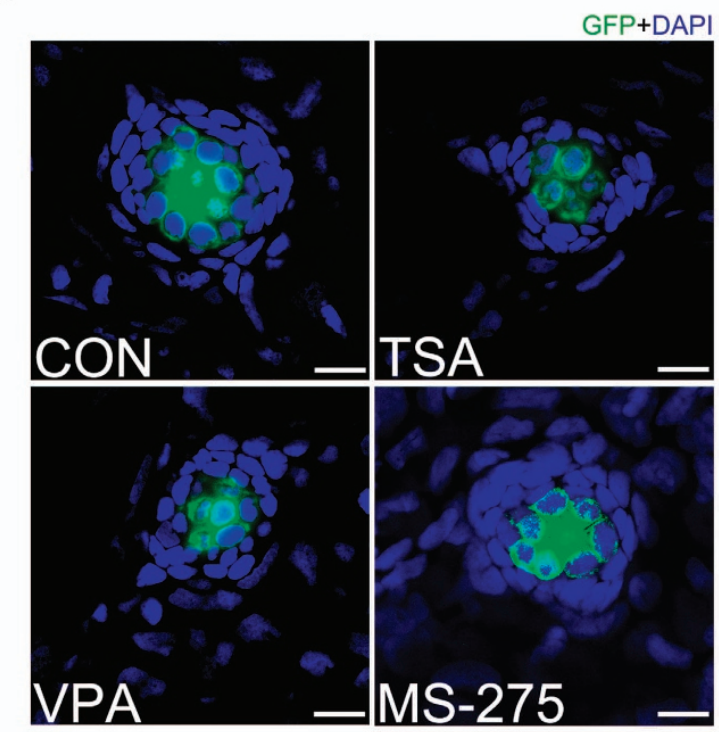

c
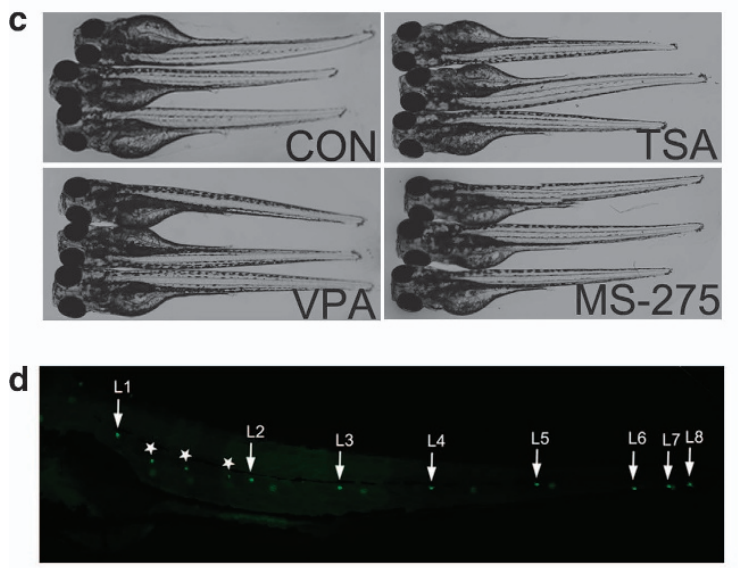

b
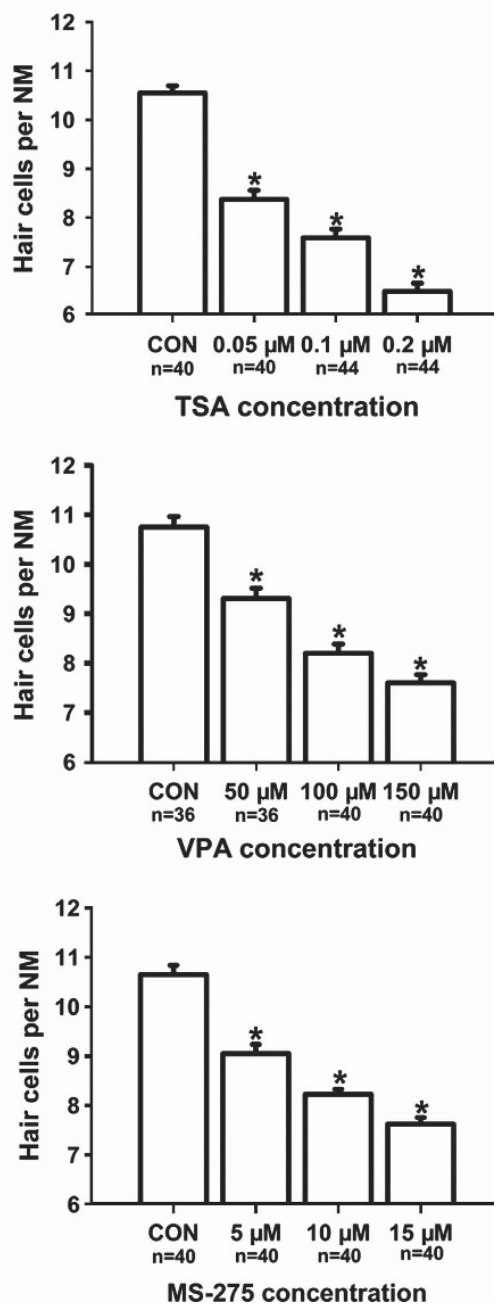

Figure 1 Detection of neuromast HCs in $5 \mathrm{dpf}$ larvae. (a) Confocal images of neuromasts from a $5 \mathrm{dpf}$ control larva and $5 \mathrm{dpf}$ larvae treated with $0.1 \mu \mathrm{m}$ TSA, $100 \mu \mathrm{m}$ VPA or $10 \mu \mathrm{m}$ MS-275. The number of neuromast HCs was assessed by GFP visualization in the transgenic line $\mathrm{Tg}(\mathrm{Brn3c}$ :mGFP). There is a significant decrease in $\mathrm{HC}$ number in the larvae treated with HDAC inhibitors. HCs are green and nuclei stained with DAPI are blue. Scale bar $=10 \mu \mathrm{m}$. (b) The average number of HCs per neuromast (NM) in $5 \mathrm{dpf}$ zebrafish larvae. The number of GFP-positive cells decreased in larvae treated with HDAC inhibitors. The HCs of the first four neuromasts along the body, L1 to L4, were recorded on one side of each fish. Bars are mean \pm s.e.m. and $n=$ total number of neuromasts. ${ }^{*} P<0.05$. (c) The zebrafish larvae treated with $0.1 \mu \mathrm{M}$ TSA, $100 \mu \mathrm{m}$ VPA or $10 \mu \mathrm{m}$ MS-275 from $3 \mathrm{dpf}$ to $5 \mathrm{dpf}$ and control larvae were morphologically normal. (d) Superimposition of a fluorescent image of a $5 \mathrm{dpf}$ zebrafish larva with neuromast HCs labeled with GFP. White arrows point to primary neuromasts L1 to L8 on the left side of the larva and the image shows secondary neuromasts $\left({ }^{*}\right)$ and a few neuromasts from the right side of the larva (not labeled).

real-time PCR using SYBR Green Master Mix and the following primer pairs: p21 forward 5'-CCTACGTTCACTCGGTAATGG-3', reverse 5'-TGGAAGTCTCCGCCCTCT-3'; p27 forward 5'-CGGGAAT CACGACTGTAGG-3' , reverse $5^{\prime}$-TGTGGGTGTCGGACTCAAT- ${ }^{\prime}$; p53 forward 5'-AAGAACAGCCTCAGCCATCC- $3^{\prime}$, reverse 5'-CAGGT CCGGTGAATAAGTGC-3'; GAPDH forward $5^{\prime}$-TGTAAGCAATGCC TCCTG-3'; reverse 5'-CATCAACGGTCTTCTGTG-3'. The amount of mRNA was calculated using GAPDH as the endogenous control.

\section{TUNEL staining}

Apoptotic cells in neuromasts were indicated by pyknotic nuclei after DAPI staining and detected by TUNEL (terminal deoxynucleotidyl transferase-mediated dUTP nick end labeling) assay as described previously. ${ }^{18}$ After washing three times with PBS, the embryos were labeled with DAPI to visualize the nuclei in the neuromasts.

\section{RESULTS}

Fewer HCs develop after exposure to HDAC inhibitors

To investigate the role of HDACs in neuromast development, we treated zebrafish larvae with TSA from $3 \mathrm{dpf}$ to $5 \mathrm{dpf}$, the time during which HCs form and begin to function. Specific labeling of HCs was confirmed using the transgenic zebrafish line Brn3c:mGFP that expresses GFP in differentiated HCs (Supplementary Figure S1). We found that $3 \mathrm{dpf}$ control larvae 
harbor $5.6 \pm 0.24$ GFP-positive HCs per neuromast $(n=40$ neuromasts), while the number of GFP-positive HCs in neuromasts of $5 \mathrm{dpf}$ larvae was significantly reduced in the presence of $0.1 \mu \mathrm{M}$ TSA compared with controls (Figures 1a and $b$; control larvae harbor $10.6 \pm 0.15$ HCs per neuromast, $n=40 \quad$ neuromasts; $0.1 \mu \mathrm{M} \quad$ TSA-treated larvae harbor $7.6 \pm 0.18$ HCs per neuromast, $n=44$ neuromasts; $P<0.05$ ). To confirm that the decrease in hair cell number induced by TSA was related to an inhibitory effect on HDACs, we performed the same experiment with the HDAC inhibitors VPA and MS-275. A reduction in HCs was also observed with both VPA (Figures 1a and b; control larvae harbor $10.8 \pm 0.22$ HCs per neuromast, $n=36$ neuromasts; $100 \mu \mathrm{M}$ VPA-treated larvae harbor $8.2 \pm 0.18 \mathrm{HCs}$ per neuromast, $n=40$ neuromasts; $P<0.05$ ) and MS-275 (Figures 1a and b; control larvae harbor $10.7 \pm 0.19$ HCs per neuromast, $n=40$ neuromasts; $10 \mu \mathrm{M}$ MS-275-treated larvae harbor $8.2 \pm 0.1 \mathrm{HCs}$ per neuromast, $n=40$ neuromasts; $P<0.05)$. At these doses, zebrafish larvae showed no evidence of morphological abnormalities suggesting that the larvae did not suffer any drug toxicity (Figure 1c). To further assess the concentration effect, we treated larvae with TSA, VPA and MS-275 at concentrations ranging from $0.05 \mu \mathrm{M}$ to $0.2 \mu \mathrm{M}, 50 \mu \mathrm{M}$ to $150 \mu \mathrm{M}$ and $5 \mu \mathrm{M}$ to $15 \mu \mathrm{M}$, respectively. We maintained the treatment for 2 days and counted the number of GFP-positive HCs in the neuromasts. Our data showed that HDAC inhibitors reduced the number of HCs in a dose-dependent manner (Figure 1b).

The effect of TSA on the development of HCs was also confirmed by the reduction of FM1-43FX staining in $5 \mathrm{dpf}$ larvae, which suggested a decrease in the number of functional $\mathrm{HCs}$ in the neuromasts (Figures $2 \mathrm{a}$ and $\mathrm{b}$ ). There were $5.7 \pm 0.24$ stained HCs in the $0.1 \mu \mathrm{M}$ TSA-treated neuromasts, which was in clear contrast to the $8.7 \pm 0.39$ HCs per neuromast in control larvae $(P<0.001)$. TSA treatment reduced the number of functional $\mathrm{HCs}$ in a dose-dependent manner, and the average number of FM1-43FX-stained HCs within each neuromast ranged from $6.0 \pm 0.38$ to $4.8 \pm 0.28$ depending on the concentration of TSA (Figure 2b). These results suggest that normal neuromast $\mathrm{HC}$ development is disrupted by the presence of HDAC inhibitors. To further distinguish functional from immature $\mathrm{HCs}$, we labeled $5 \mathrm{dpf}$ Brn3c:mGFP transgenic control and TSA-treated $(0.1 \mu \mathrm{M})$ fish with FM1-43FX, and no difference in the number of FM1$43 \mathrm{FX}+/ \mathrm{GFP}+$ cells were observed between the two conditions (Supplementary Figure S2; $n=25$ neuromasts observed for each condition; $P=0.196$ )

\section{Fewer SCs are formed after exposure to HDAC inhibitors} The SCs were identified with an antibody against Sox2, which is highly expressed in the SCs of the neuromasts. We counted the Sox2-labeled cells in the neuromasts after treatment with different concentrations of the HDAC inhibitors and observed a dose-dependent reduction in the number of stained SCs (Figure 3). These results suggest from another perspective that HDAC activity is involved in neuromast development. To confirm that the deficiency in neuromast development was, a
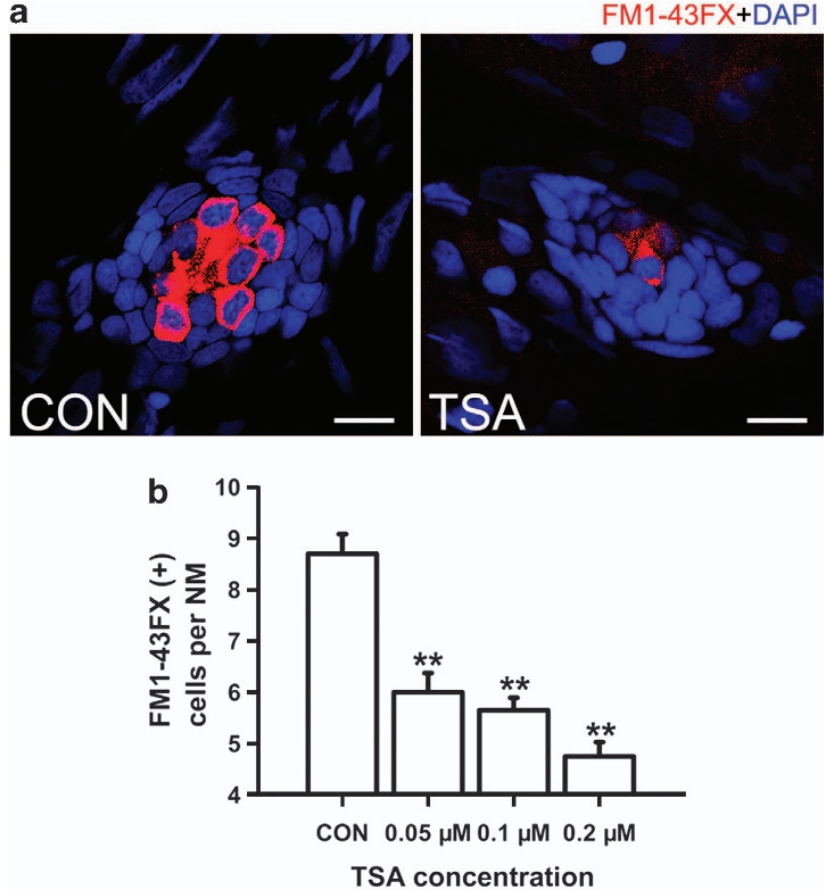

Figure 2 Detection of neuromast hair cells with live staining (FM1$43 F X$ ) in $5 \mathrm{dpf}$ larvae. (a) Confocal images of neuromasts from a control and $0.1 \mu \mathrm{m}$ TSA-treated larva at $5 \mathrm{dpf}$ that have been labeled for FM1-43FX to detect functional HCs. Fewer FM1-43FXpositive cells developed in the TSA-treated larva compared with the untreated control. Nuclei are stained with DAPI and scale bars $=10 \mu \mathrm{m}$. (b) The average number of functional HCs per neuromast (NM) in $5 \mathrm{dpf}$ zebrafish larvae following TSA treatment. $* * P<0.001$.

indeed, caused by the inhibition of HDAC activity, we examined the level of acetylated (Ace) histone $\mathrm{H} 3$ and $\mathrm{H} 4$ by western blot (Figure 4a). Our data showed that HDAC inhibitors caused a significant increase in the levels of both Ace $\mathrm{H} 3$ and Ace $\mathrm{H} 4$ compared with controls. To more accurately localize Ace $\mathrm{H} 3 / \mathrm{H} 4$ expression to the specific cell types in the neuromast, we performed immunostaining against Myosin VI, which is expressed in differentiated HCs. Visualization with both Myosin VI and Ace $\mathrm{H} 3 / \mathrm{H} 4$ labeling shows that Ace $\mathrm{H} 3 / \mathrm{H} 4$-positive cells are found outside the centrally located rosette of HCs with little or no overlap between them in either the HDAC inhibitor-treated group or controls (Figure $4 \mathrm{~b}$ ). To determine the extent of overlap between Ace $\mathrm{H} 3 / \mathrm{H} 4$ protein expression and neuromast SCs, we immunostained for Sox2 protein expression. In the $5 \mathrm{dpf}$ HDAC inhibitor-treated embryos, we observed a coincidence between the expression of both markers indicating that the elevated Ace $\mathrm{H} 3 / \mathrm{H} 4$ protein expression is mainly present in SCs (Supplementary Figure S3). This observation provided evidence that hyperacetylated histones in SCs upon inhibitor treatment might indeed be responsible for the decreased cell number in neuromasts.

\section{HDAC inhibitors affect neuromast cell proliferation}

To study the effect of HDAC inhibitors on cell proliferation in neuromasts in the lateral line of zebrafish, we incubated $3 \mathrm{dpf}$ 

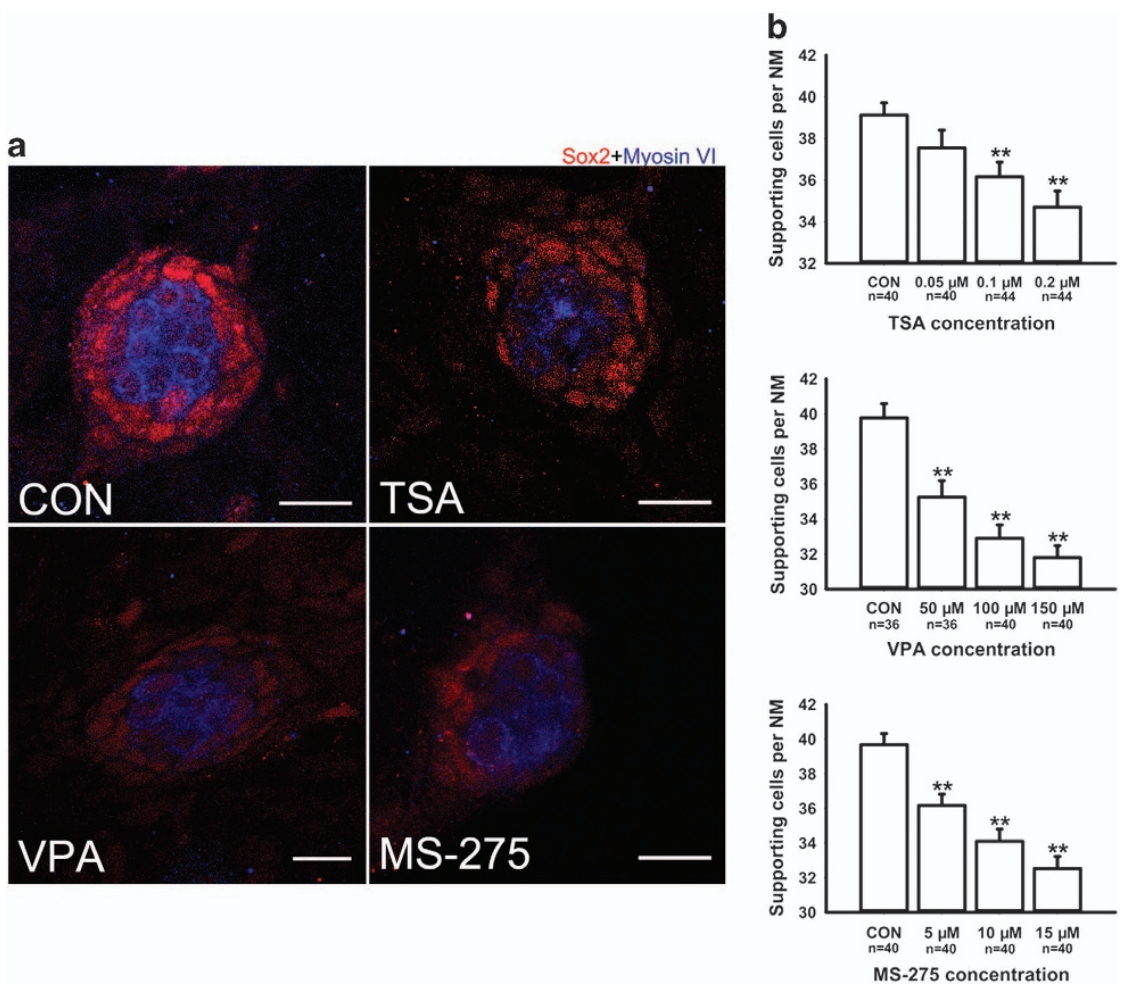

Figure 3 Detection of SCs with Sox2 in $5 \mathrm{dpf}$ larvae. (a) Confocal images of neuromasts from a control larva and larvae treated with $0.1 \mu \mathrm{m} \mathrm{TSA}, 100 \mu \mathrm{m}$ VPA or $10 \mu \mathrm{m}$ MS-275 at $5 \mathrm{dpf}$ that have been labeled by Sox2 to detect SCs. Fewer Sox2-positive cells develop in the neuromasts of larvae treated with HDAC inhibitors. HCs are stained with myosin VI (blue), and red spots represent the Sox2 signal. Scale bars $=10 \mu \mathrm{m}$. (b) The average number of SCs per neuromast (NM) in $5 \mathrm{dpf}$ zebrafish larvae. The number of Sox2- positive cells decreased in larvae treated with HDAC inhibitors. The SCs of the first four neuromasts along the body, L1 to L4, were recorded on one side of each fish. Bars are mean \pm s.e.m. and $n=$ total number of neuromasts. ${ }^{* *} P<0.001$.

zebrafish larvae with both BrdU and VPA, TSA or MS-275 for 2 days (Figure 5a). The level of neuromast cell proliferation was determined by counting the number of BrdU-positive cells in the L1-L4 neuromasts. The BrdU index was calculated as the ratio of BrdU-positive nuclei to total DAPI-stained nuclei. The results are quantified in Figure 5b. For $0.1 \mu \mathrm{M}$ TSA, $100 \mu \mathrm{M}$ VPA and $10 \mu \mathrm{M}$ MS-275, the control neuromasts had a BrdU index of $0.237 \pm 0.007,0.247 \pm 0.009$ and $0.23 \pm 0.009$, respectively, and the treated neuromasts had a BrdU index of $0.17 \pm 0.005, \quad 0.169 \pm 0.006$ and $0.16 \pm 0.007$, respectively ( $n=36$ neuromasts and $P<0.001$ for all experiments).

Measuring both Myosin VI and BrdU labeling in neuromasts of $5 \mathrm{dpf}$ larvae allowed us to distinguish the newly differentiated HCs from cell proliferation. Our analysis revealed that in inhibitor-treated neuromasts, the BrdU incorporation was mainly detected in the periphery of the neuromast and there was very little overlap of signals. In control fish, however, a considerable amount of Myosin VIexpressing cells co-labeled with BrdU (Figures $5 \mathrm{c}$ and d; larvae treated with $0.1 \mu \mathrm{M}$ TSA harbor $1.44 \pm 0.23$ Myosin VI-positive and BrdU-positive cells per neuromast compared with $3.36 \pm 0.25$ cells in the control larvae, $n=36$ neuromasts; $P<0.001$. Larvae treated with $100 \mu \mathrm{M}$ VPA harbor $1.97 \pm 0.22$ Myosin VI-positive and BrdU-positive cells per neuromast compared with $3.83 \pm 0.16$ cells in the control larvae, $n=36$ neuromasts; $P<0.001$. Larvae treated with $10 \mu \mathrm{M}$ MS-275 harbor $1.5 \pm 0.18$ Myosin VI-positive and BrdU-positive cells per neuromast compared with $4.03 \pm 0.19$ cells in the control larvae, $n=36$ neuromasts; $P<0.001$ ).

We further counted the cells that are double labeled with anti-Sox 2 and anti-BrdU antibodies. We confirmed that the majority of BrdU-positive cells also express Sox2 in both control and TSA-treated neuromasts. The average numbers of BrdU-positive cells that also express the Sox 2 protein in control and $0.1 \mu \mathrm{M}$ TSA-treated fish are $8.92 \pm 0.5$ cells and $6.11 \pm 0.21$ cells per neuromast, respectively $(P<0.001)$ (Figure 5e). Similar results were also obtained in VPA- and MS-275-treated groups (control larvae harbor $7.5 \pm 0.36$ BrdU-positive SCs per neuromast, $n=36$ neuromasts; $100 \mu \mathrm{M}$ VPA treated-larvae harbor $5.19 \pm 0.27$ BrdU-positive SCs per neuromast, $n=36$ neuromasts; $P<0.001$. Control larvae harbor $7.39 \pm 0.33$ BrdU-positive SCs per neuromast, $n=36$ neuromasts; $10 \mu \mathrm{M}$ MS-275 treated-larvae harbor $5.33 \pm 0.26$ BrdU-positive SCs per neuromast, $n=36$ neuromasts; $P<0.001$ ). These findings suggest that HDAC inhibitors significantly decrease the percentage of BrdU-positive cells indicating that there are fewer cells progressing into the S-phase of the cell cycle.

On the basis of the BrdU uptake studies, we further focused on the changes in p21 and p27 and sought to determine 


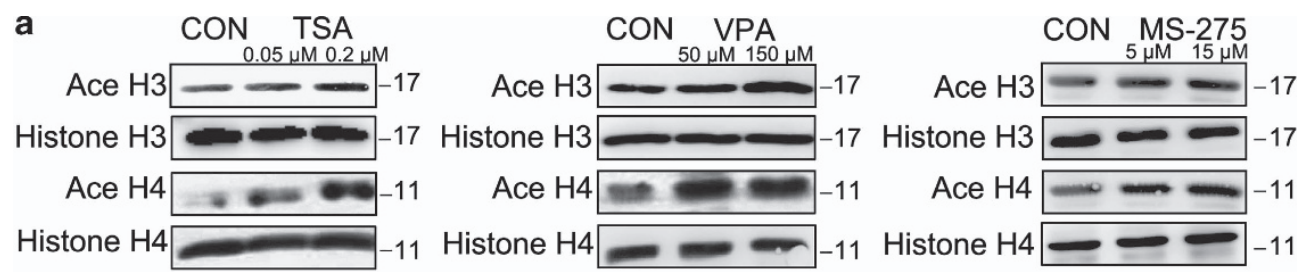

b
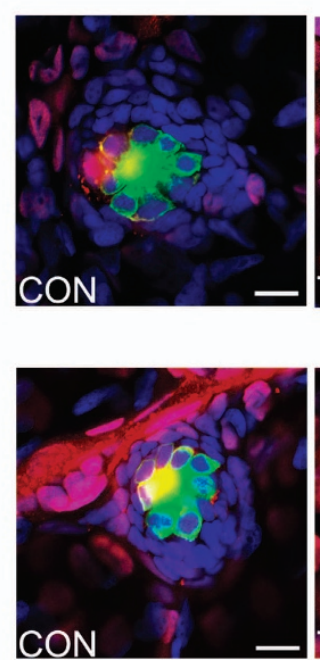
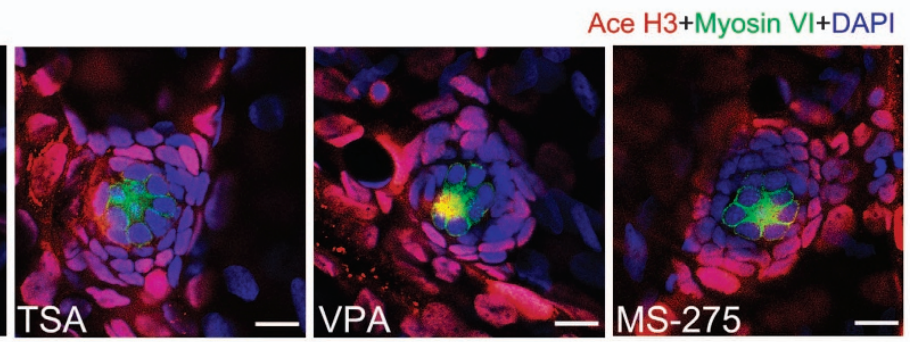

Ace $\mathrm{H} 4+\mathrm{Myosin} \mathrm{VI}+\mathrm{DAPI}$
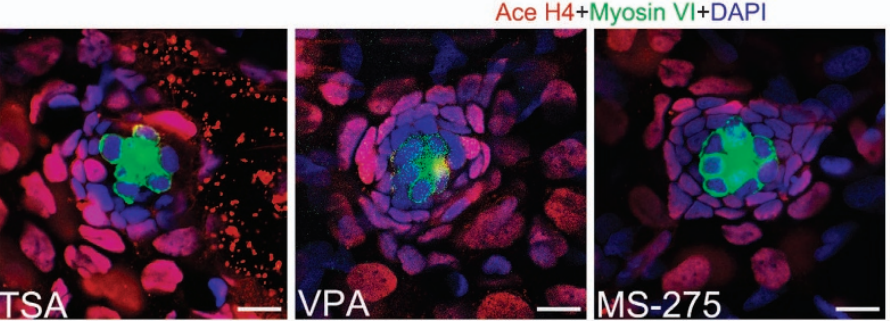

Figure 4 The effect of HDAC inhibitors on the levels of histone acetylation. (a) Western blot analysis of protein extracts from $5 \mathrm{dpf}$ control larvae and larvae treated with HDAC inhibitors for 2 days starting at $3 \mathrm{dpf}$. HDAC inhibitors induced the accumulation of acetylated (Ace) $\mathrm{H} 3$ and $\mathrm{H} 4$. Histones $\mathrm{H} 3$ and $\mathrm{H} 4$ were included as controls. (b) Confocal images of neuromasts from a $5 \mathrm{dpf}$ control larva and from $5 \mathrm{dpf}$ larvae treated with TSA, VPA or MS-275. Larvae were treated with $0.1 \mu \mathrm{m}$ TSA, $100 \mu \mathrm{m}$ VPA or $10 \mu \mathrm{m}$ MS-275 for 2 days starting at 3 dpf and were stained (red) by antibodies against Ace histone $\mathrm{H} 3$ and Ace histone $\mathrm{H} 4$. The levels of Ace histone $\mathrm{H} 3$ and Ace histone $\mathrm{H} 4$ are increased in the presence of HDAC inhibitors compared with the controls (CON). HCs were labeled with antibodies against Myosin VI (green) and nuclei were stained with DAPI (blue). Scale bar $=10 \mu \mathrm{m}$.

whether the expression of these cell cycle regulators differed between control and TSA-treated larvae. Real-time RT-PCR showed that expressions of both p21 and p27 mRNA were increased in embryos treated with TSA (Figure 5f). We also examined the possible role of $\mathrm{p} 53$. We found that, although the level of p53 was only slightly increased in $0.1 \mu \mathrm{M}$ TSA-treated larvae, it increased almost threefold in embryos treated with $0.2 \mu \mathrm{M}$ TSA (Figure 5f).

High concentrations of TSA induce apoptosis in neuromast cells

HDACs have been shown to be involved in regulating cell proliferation and programmed cell death. To assess the effect of HDAC inhibition on apoptosis in neuromasts, we quantified cell death by counting the number of pyknotic nuclei (a hallmark of apoptosis) in the larval neuromasts. Neuromast cells treated with $0.2 \mu \mathrm{M}$ TSA contained a slightly increased number of pyknotic nuclei compared with control larvae and those treated with low concentrations of TSA. We found $0.19 \pm 0.15$ pyknotic nuclei per neuromast ( $n=20$ neuromasts) in control larvae and $0.57 \pm 0.18$ pyknotic nuclei per neuromast $(n=20$ neuromasts, $P<0.05)$ in fish treated with $0.2 \mu \mathrm{M}$ TSA. Most of the cells with pyknotic nuclei were positively stained in a TUNEL assay (Figure 6a). Moreover, pyknotic cells in neuromasts following $0.2 \mu \mathrm{M}$ TSA treatment were also immunohistochemically positive for cleaved caspase-3 (Figure 6b). These observations suggest that high concentrations of HDAC inhibitors induce cell death in neuromasts.

\section{DISCUSSION}

In the present study, we have shown a requirement for HDAC activity in the formation of neuromasts in the zebrafish lateral line. Treatment of embryos with HDAC inhibitors was initiated at $3 \mathrm{dpf}$ because it has been previously reported that lateral line neuromasts in the zebrafish embryo enter a phase of maturation with functional HCs appearing at $3 \mathrm{dpf}^{3,19}$ Thus, the period between $3 \mathrm{dpf}$ and $5 \mathrm{dpf}$ is critical for the study of HC development. We found that pharmacological HDAC inhibition led to a significant reduction in the number of $\mathrm{HCs}$ in Brn3c:mGFP transgenic embryos. FM1-43FX staining was used to further distinguish fully differentiated (functional) HCs from immature HCs. When we labeled $5 \mathrm{dpf}$ Brn3c:mGFP transgenic fish with FM1-43FX, we observed no difference in the number of FM1-43FX + /GFP + cells in control and TSA-treated groups. This indicated that HDAC activity might not have functions at the late stages of HC differentiation when they acquire mechanotransduction function. The HDAC inhibitor treatments also reduced the 
a

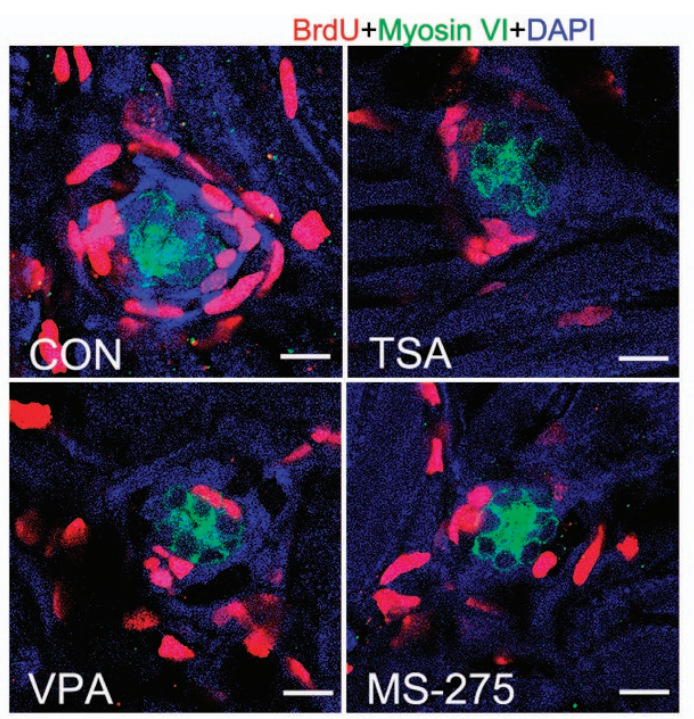

b
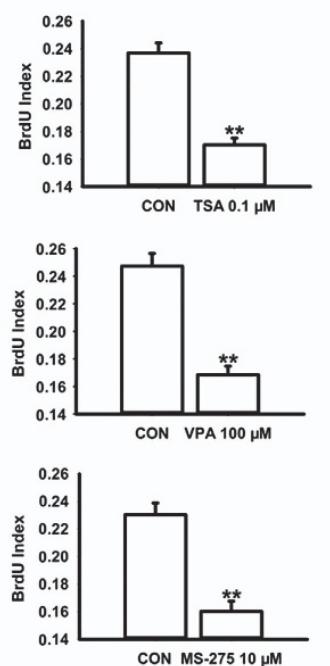

C

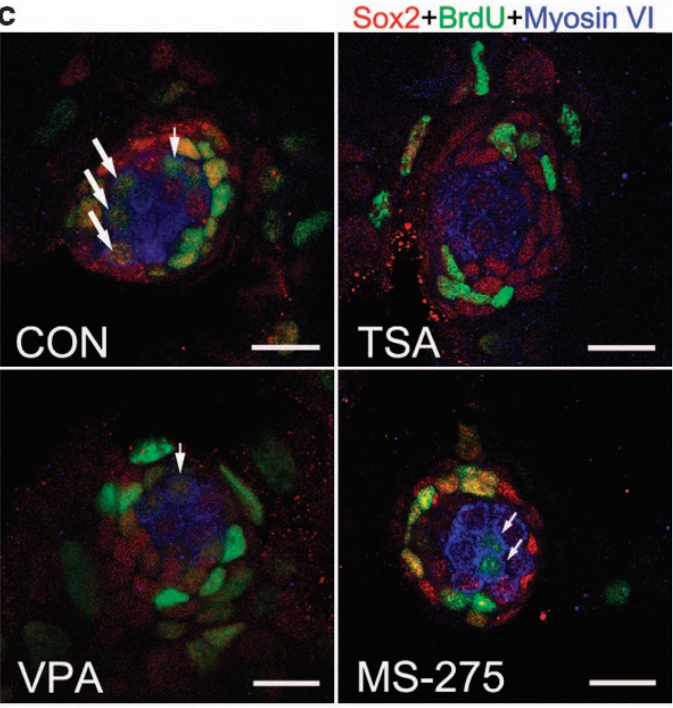

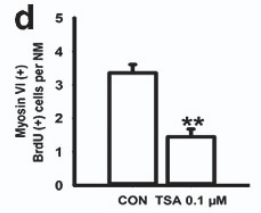
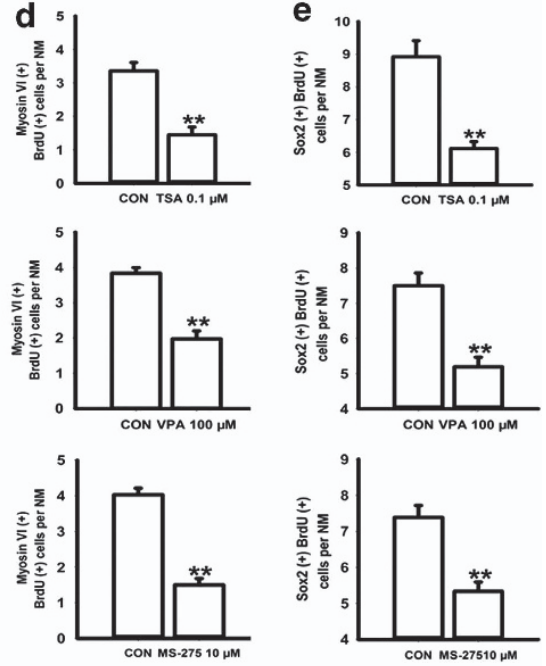

f

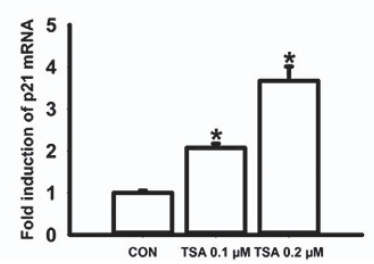

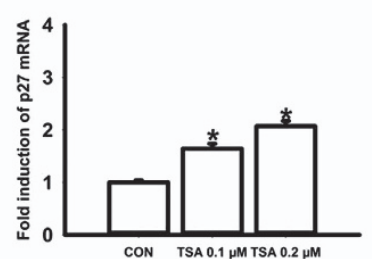

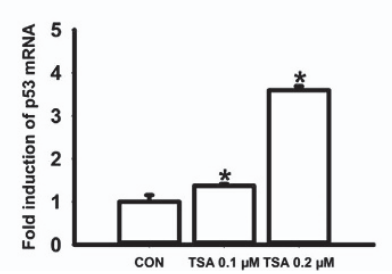

Figure 5 HDAC inhibitors inhibit neuromast cell proliferation. (a) Confocal images of neuromasts from a $5 \mathrm{dpf}$ control and from $5 \mathrm{dpf}$ embryos treated with $0.1 \mu \mathrm{m}$ TSA, $100 \mu \mathrm{m}$ VPA or $10 \mu \mathrm{m}$ MS-275 that have been labeled by BrdU to detect proliferating cells. The number of BrdU-labeled cells is much larger in control than in HDAC inhibitor-treated embryos. Red spots represent BrdU signal. HCs are labeled with an antibody against myosin VI (green). Scale bar $=10 \mu \mathrm{m}$. (b) Quantification of replicating cells in the neuromasts for each experimental condition. The BrdU index is higher in the control relative to the HDAC inhibitor-treated fish. The proliferating cells of the first four neuromasts along the body, $L 1$ to $L 4$, were recorded on one side of each fish. Bars are mean \pm s.e.m. and $n=36$ neuromasts per condition. ${ }^{*} P<0.001$. (c) Confocal images of neuromasts from a $5 \mathrm{dpf}$ control and from $5 \mathrm{dpf}$ embryos treated with $0.1 \mu \mathrm{m}$ TSA, $100 \mu \mathrm{m}$ VPA or $10 \mu \mathrm{m}$ MS-275. HDAC inhibitor treatment decreased the numbers of both BrdU-positive HCs and BrdU-positive SCs in neuromasts. White arrows indicate HCs that were derived from proliferating SCs. Scale bar $=10 \mu \mathrm{m}$. (d, e) Quantification of replicating cells co-labeled with Myosin VI or Sox2 per neuromast (NM) in control and HDAC inhibitor-treated larvae. Bars are mean \pm s.e.m. and $n=36$ neuromasts per condition. ${ }^{* *} P<0.001$. (f) Real-time RT-PCR analysis showed that TSA treatment increases the mRNA expression of p21, p27 and p53. The bars represent the means \pm s.d. of the ratios of p21, p27 and p53 to GAPDH in the real-time RT-PCR data. ${ }^{*} P<0.05$. 
a

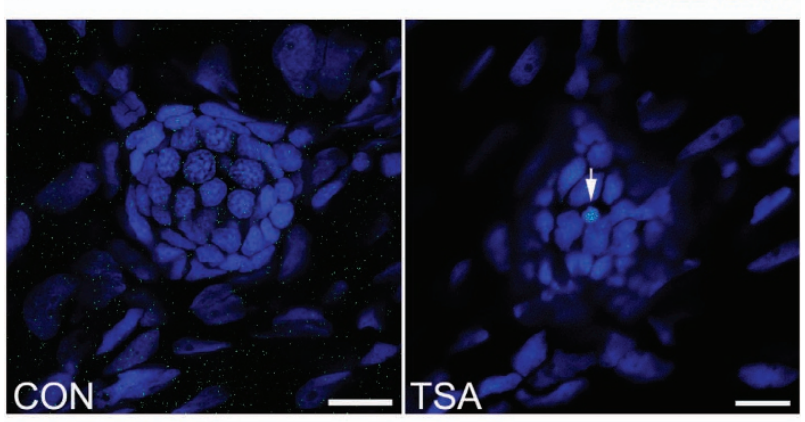

b

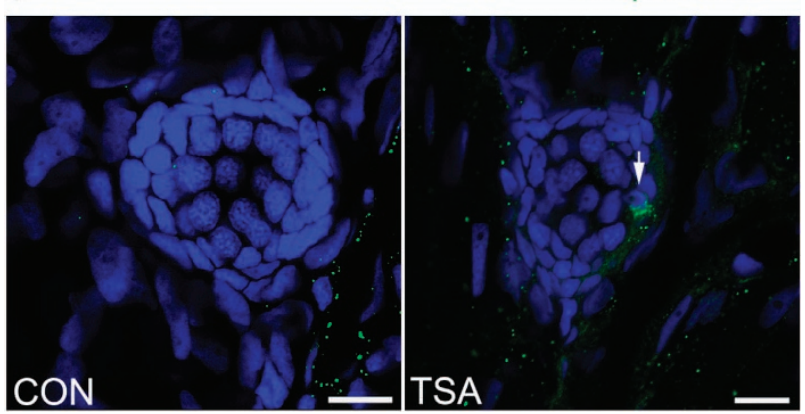

Figure $6 \mathrm{~A}$ high concentration of TSA $(0.2 \mu \mathrm{m})$ results in apoptotic cells within the lateral line neuromast. (a) TUNEL staining in the neuromast from a control and TSA-treated larva at $5 \mathrm{dpf}$. White arrows indicate TUNEL-positive cells. (b) Cleaved caspase-3 staining in the neuromast from a control and TSA-treated larva. White arrows indicate cells with cleaved caspase-3. Scale bar $=10 \mu \mathrm{m}$.

number of SCs, which have been suggested to be the permanent source of newly differentiated HCs. Taken together, these data suggest that HDAC activity is required for the development of neuromasts in the zebrafish lateral line.

The observed difference in the numbers of HCs and SCs between HDAC inhibitor-treated and untreated larvae can be explained by a decrease in cell proliferation and/or an increase in cell death. We first evaluated cell proliferation in neuromasts upon treatment with HDAC inhibitors. It is well known that neuromast HCs continue to be renewed and that this probably occurs throughout the entire life of zebrafish larvae. The renewal of $\mathrm{HCs}$ is suggested to come mainly from the continuous proliferation of the SCs. ${ }^{20}$ Our observations showed that HDAC inhibitors significantly reduced the BrdU index, and this anti-proliferative effect is consistent with the known function of HDAC inhibitors as key regulators in reducing the proliferation of tumor cells. Thus, our data suggest that HDAC activity is required for neuromast cell proliferation.

It has been previously observed that the histone hyperacetylation induced by HDAC inhibitors is responsible for the anti-proliferative effect, and this is probably through selective alteration of the expression of genes involved in G1 to S-phase progression. ${ }^{21}$ Much effort has been devoted to analyzing the coordinated interaction between cyclins and cyclin-dependent kinase (CDK) complexes. Cyclin-dependent kinase inhibitors (CKI), such as those of the Cip/Kip family (p21, p27, and p57), can inhibit a broad range of cyclin/Cdk complexes and cause cell-cycle arrest. Upregulation of CKI results in the inhibition of cell cycle progression in the G1 phase, and downregulation of CKI is required for re-entry into S-phase of the cell cycle. Treatment with TSA led to increased expression of the CDK inhibitors p21 and p27, and this suggests that p21 and p27, and p21 in particular, might be relevant in mediating the antiproliferative effect of HDAC inhibitors on neuromast cells. This is in agreement with previous studies showing that almost all HDAC inhibitors induce a significant increase in the expression of $\mathrm{p} 21 .{ }^{22}$

To analyze the possibility of increased cell death as another explanation for the reduction in cell numbers, we determined the effect of TSA treatment on cell death in neuromasts. We found that cell death is quite rare in both control neuromasts and those treated with low concentrations of TSA. This is in agreement with previous findings showing that the neuromasts in zebrafish undergo constant turnover. ${ }^{23}$ However, we observed that treatment with a higher concentration of TSA than that needed for inhibiting proliferation leads to slightly increased apoptosis. It has been shown that activation of p53 can lead to cell cycle arrest and apoptosis. We further examined the expression of p53 mRNA and observed a significant upregulation in mRNA level when the larvae were treated with $0.2 \mu \mathrm{M}$ TSA. Our findings suggest that the induction of apoptosis is related to HDAC deficiency, and the effect of high concentration of TSA treatment on neuromast apoptosis is partly activated through a p53-dependent pathway.

Other factors shown to be involved in prosensory development in the neuromast include Fgf and Notch signaling pathways. ${ }^{19}$ These signaling pathways are required for sensory precursor specification. The Notch-Delta signaling pathway has been shown previously to be involved in HC formation through a mechanism of lateral inhibition. ${ }^{24}$ Notch signaling limits the number of HCs and determines the ratio of HCs to other neuromast cell types, and a failure in this signaling is associated with ectopic differentiation of excess HCs. ${ }^{24} \mathrm{~A}$ recent study in zebrafish showed that HDAC 1 is required to maintain vertebrate neurogenesis via repression of Notch target gene expression. ${ }^{10}$ Therefore, future experiments will focus on whether the effect of HDACs on HC differentiation is through a direct or indirect interaction with members of signaling pathways such as the Notch-Delta signaling pathway. We will examine not only the levels of gene expression of the signaling pathway proteins but also the proneural gene expression in larvae treated with HDAC inhibitors. Gene knockdown analysis has suggested that several proneural genes have an effect on the determination or differentiation of $\mathrm{HCs}$ in the zebrafish lateral line neuromasts. ${ }^{25,26}$ These proneural genes, such as Atoh1, interact with the Delta/Notch signaling pathway and this confirms their early proneural function. ${ }^{27,28}$ The precise mechanism that ensures the development of HCs in 
numbers proportional to the size of the neuromasts will require further analysis.

The present study shows that HDAC activity is involved in the development of HCs and SCs in the neuromasts of the zebrafish lateral line. This might be through the regulation of cell proliferation in the neuromasts. HDAC inhibitors exhibit antiproliferative activity and slightly induce apoptosis at high concentrations. These events are accompanied by the induction of p21, p27 and p53. Future studies are needed to determine the specific effect of different HDACs and to identify the exact mechanisms involved in the regulation of CKIs.

\section{ACKNOWLEDGEMENTS}

This work was supported by grants from the Major State Basic Research Development Program of China (973 Program) (2011CB504506, 2010CB945503), the National Natural Science Foundation of China (Nos. 81230019, 81070793, 81371094, 81300825), the Program for Changjiang Scholars and Innovative Research Team in Universities (IRT1010), the Medical Guiding Fund of the Science and Technology Commission of Shanghai Municipality (10411962100), the Specialized Research Fund for the Doctor Program of Higher Education (20120071110077), the Innovation Program of Major Basic Research Project, the Shanghai RisingStar Program (12QA1400500), the Science and Technology Commission of Shanghai Municipality (09DJ1400602) and the Program of Leading Medical Personnel in Shanghai. We thank Professor Min Yu, Jingying $\mathrm{Hu}$ and Shaoyang Sun for their expert fish care, and Yalin Huang for her technical assistance with confocal microscopy. We thank Professor Zhengyi Chen for his generous gift of the Brn3c:mGFP transgenic line.

1 Montgomery J, Carton G, Voigt R, Baker C, Diebel C. Sensory processing of water currents by fishes. Philos Trans R Soc Lond B Biol Sci 2000; 355: 1325-1327.

2 Nicolson T. The genetics of hearing and balance in zebrafish. Annu Rev Genet 2005; 39: 9-22.

3 Raible DW, Kruse GJ. Organization of the lateral line system in embryonic zebrafish. J Comp Neurol 2000; 421: 189-198.

4 Jones JE, Corwin JT. Regeneration of sensory cells after laser ablation in the lateral line system: hair cell lineage and macrophage behavior revealed by time-lapse video microscopy. J Neurosci 1996; 16: 649-662.

5 Owens KN, Santos F, Roberts B, Linbo T, Coffin AB, Knisely AJ et al. Identification of genetic and chemical modulators of zebrafish mechanosensory hair cell death. PLoS Genet 2008; 4: e1000020.

6 Berger SL. Histone modifications in transcriptional regulation. Curr Opin Genet Dev 2002; 12: 142-148.

7 Kouzarides T. Chromatin modifications and their function. Cell 2007; 128: 693-705.

8 Provenzano MJ, Domann FE. A role for epigenetics in hearing: Establishment and maintenance of auditory specific gene expression patterns. Hear Res 2007; 233: 1-13.

9 Haberland M, Montgomery RL, Olson EN. The many roles of histone deacetylases in development and physiology: implications for disease and therapy. Nat Rev Genet 2009; 10: 32-42.
10 Cunliffe VT. Histone deacetylase 1 is required to repress Notch target gene expression during zebrafish neurogenesis and to maintain the production of motoneurones in response to hedgehog signalling. Development 2004; 131: 2983-2995.

11 Harrison MR, Georgiou AS, Spaink HP, Cunliffe VT. The epigenetic regulator Histone Deacetylase 1 promotes transcription of a core neurogenic programme in zebrafish embryos. BMC Genomics 2011; 12: 24.

12 Montgomery RL, Hsieh J, Barbosa AC, Richardson JA, Olson EN. Histone deacetylases 1 and 2 control the progression of neural precursors to neurons during brain development. Proc Natl Acad Sci USA 2009; 106: 7876-7881.

13 Marchion D, Munster P. Development of histone deacetylase inhibitors for cancer treatment. Expert Rev Anticancer Ther 2007; 7: 583-598.

14 Chuang DM, Leng Y, Marinova Z, Kim HJ, Chiu CT. Multiple roles of HDAC inhibition in neurodegenerative conditions. Trends Neurosci 2009; 32: 591-601.

15 Gottesfeld JM, Pandolfo M. Development of histone deacetylase inhibitors as therapeutics for neurological disease. Future Neurol 2009; 4: 775-784.

16 Slattery EL, Speck JD, Warchol ME. Epigenetic influences on sensory regeneration: histone deacetylases regulate supporting cell proliferation in the avian utricle. J Assoc Res Otolaryngol 2009; 10: 341-353.

17 Kimmel CB, Ballard WW, Kimmel SR, Ullmann B, Schilling TF. Stages of embryonic development of the zebrafish. Dev Dyn 1995; 203: 253-310.

18 Cole LK, Ross LS. Apoptosis in the developing zebrafish embryo. Dev Biol 2001; 240: 123-142.

19 Ma EY, Raible DW. Signaling pathways regulating zebrafish lateral line development. Curr Biol 2009; 19: R381-R386.

20 Hernandez PP, Olivari FA, Sarrazin AF, Sandoval PC, Allende ML. Regeneration in zebrafish lateral line neuromasts: expression of the neural progenitor cell marker sox2 and proliferation-dependent andindependent mechanisms of hair cell renewal. Dev Neurobiol 2007; 67: 637-654.

21 Dalvai M, Bystricky K. The role of histone modifications and variants in regulating gene expression in breast cancer. J Mammary Gland Biol Neoplasia 2010; 15: 19-33.

22 Ocker M, Schneider-Stock R. Histone deacetylase inhibitors: signalling towards p21cip1/waf1. Int J Biochem Cell Biol 2007; 39: 1367-1374.

23 Williams JA, Holder N. Cell turnover in neuromasts of zebrafish larvae. Hear Res 2000; 143: 171-181.

24 Haddon C, Jiang YJ, Smithers L, Lewis J. Delta-Notch signalling and the patterning of sensory cell differentiation in the zebrafish ear: evidence from the mind bomb mutant. Development 1998; 125: 4637-4644.

25 Sarrazin AF, Villablanca EJ, Nunez VA, Sandoval PC, Ghysen A, Allende $M L$. Proneural gene requirement for hair cell differentiation in the zebrafish lateral line. Dev Biol 2006; 295: 534-545.

26 Bermingham NA, Hassan BA, Price SD, Vollrath MA, Ben-Arie N, Eatock RA et al. Math1: an essential gene for the generation of inner ear hair cells. Science 1999; 284: 1837-1841.

27 Millimaki BB, Sweet EM, Dhason MS, Riley BB. Zebrafish atoh1 genes: classic proneural activity in the inner ear and regulation by Fgf and Notch. Development 2007; 134: 295-305.

28 Itoh M, Chitnis AB. Expression of proneural and neurogenic genes in the zebrafish lateral line primordium correlates with selection of hair cell fate in neuromasts. Mech Dev 2001; 102: 263-266.

This work is licensed under a Creative Commons Attribution-NonCommercial-ShareAlike 3.0 Un-
ported License. To view a copy of this license, visit http:// creativecommons.org/licenses/by-nc-sa/3.0/

Supplementary Information accompanies the paper on Experimental \& Molecular Medicine website (http://www.nature.com/emm) 